\title{
Preliminary Study on Data Fusion Based on Internet of Things of Eucalyptus Plantation
}

\author{
Deng Guang ${ }^{1, *}$, Yu Xinwen ${ }^{1}$, Fan Dongpu ${ }^{1}$, Zhu Chen ${ }^{2}$ and Zhang $x^{1}$ \\ ${ }^{1}$ Research Institute of Forest Resource Information Techniques, China Academy of Forestry Sciences, 100091 Beijing, China \\ ${ }^{2}$ Suzhou Yingchuang Kyushu Information Technology Co., Ltd., Suzhou, China
}

\begin{abstract}
Eucalyptus is the main fast-growing and high-yielding tree species in southern China and is the main source of wood production. Over the years, there have been many reports that eucalyptus is a water pump and that eucalyptus causes serious damage to understory vegetation. In this paper, a sensor network of eucalyptus plantation has been established in Yinling branch of Gaofeng Forest Farm in Guangxi, the main eucalyptus producing province. The sensor network can monitor the DBH changes, trunk runoff, soil moisture, environmental information and so on of eucalyptus in the stand in real time. The collected data are uploaded to the artificial forest Internet of Things monitoring information cloud management system established in this paper. This paper introduces the functions of the cloud management system in detail. On this basis, this paper discusses the fusion of high-frequency information collected from monitoring points to meet different data requirements. The research on data fusion in this paper is very preliminary. In the conclusion part, the future research on long-term monitoring data fusion of eucalyptus forest is prospected.
\end{abstract}

\section{Introduction}

The southeast coastal areas of China have excellent natural conditions and are suitable for the development of short-term industrial raw material forests such as eucalyptus and tropical precious timber forests. Guangxi is the first country to realize large-scale artificial afforestation and clone of eucalyptus, becoming an important eucalyptus fast-growing forest base in the country.

Internet of Things technologies such as wireless sensor networks have been applied in agriculture, orchards and other fields in recent years, but there are still many technical problems to be solved, such as the large forest area and complex environment, how to arrange the collection nodes with sensing function and how to send wireless communication methods to the server. Internet of Things technologies such as wireless sensor network, field rapid measurement and feedback are adopted to sense the information of soil moisture, soil nutrients, environment, light, forest growth and other information of the artificial timber forest in a timely manner, so as to realize fine management and improve the management level.

The existing plantation cultivation technology focuses on the seedling stage, and its Internet of Things monitoring is relatively easy, while there is still a lack of detailed technical research in the growth and management stage of trees. Traditional geographic information and remote sensing technologies focus on resource monitoring and data management over a large time span and cannot be combined with the process and stage of plantation management. Forest managers use mathematical statistics to obtain a long-term management plan, which cannot be adjusted in time according to the actual situation of water, soil and gas.

The most famous research in the world is Lindsey Rustad, an ecologist at the North Research Station of the U.S. Forest Service, who presided over the establishment of the Smart Forest Experimental Forest Network[1,2] for the $21 \mathrm{st}$ century. The network uses digital environmental sensors and remote communication technology to accurately acquire environmental data in real time in the experimental forests in the United States. Liu Yunfei of Nanjing Forestry University and others conducted research on the construction of the Internet of Things for poplar plantation ecosystem and biodiversity in Yang Shulin, Baoying County, Jiangsu Province.[4,6,8]

Traditional sensor network fusion algorithm processes a large number of original data collected by nodes in the network to remove redundant data, so as to achieve the purpose of saving energy consumption and improving the network life cycle.[3] However, doing so will inevitably lead to loss of information. In this paper, after the sensor data network data is collected and stored in the cloud, the data fusion experimental research is carried out in the application layer. The purpose of data fusion of artificial forest sensor network is to serve the purpose of eucalyptus plantation management.

\footnotetext{
Corresponding author: javawsdp@sina.com
} 


\section{Research area and data}

\subsection{Research area}

The sample area of this study is located in Yinling branch of Guangxi Gaofeng Forest Farm, which belongs to Xixiangtang District of Nanning City, Guangxi. Guangxi Gaofeng Forest Farm is characterized by sultry and humid summer, cold and snowless winter, long summer and short winter, with an average wind speed of about $1.9 \mathrm{~m} / \mathrm{s}$, belonging to subtropical monsoon climate. The annual average temperature is about 21.6 degrees, and the temperature range is between minus 2.4 degrees and 40.4 degrees above zero. The rainy season is mainly concentrated in summer with abundant rainfall, ranging from $827.9 \mathrm{~mm}$ to $1797.1 \mathrm{~mm}$, with an annual average rainfall of $1304.2 \mathrm{~mm}$. The number of days of rainfall in a year is about 155 days and the daily average rainfall is about $6.5 \mathrm{~mm}$. The amount of water evaporation in the forest area is relatively small, with the average annual evaporation basically kept at about $1736.6 \mathrm{~mm}$ and the average humidity at about $79 \%$.

The Yinling branch has an altitude of 186 meters and an area of 2,865.47 hectares. The origin of the stand in the experimental plot area is the plantation initiation in June 2015. The tree species are pure giant tail eucalyptus. The method is to remove miscellaneous irrigation by full ridge. The afforestation density is 111 trees $/ \mathrm{mu}$, and the distribution is relatively uniform. The fertilizer is applied once a year from April to May. The special fertilizer for Eucalyptus grandis with total available nutrients of nitrogen $(\mathrm{N})$, phosphorus $(\mathrm{P})$ and potassium $(\mathrm{K})$ over $30 \%$ is applied. The fertilizer application rate is controlled at $500 \mathrm{~g} / \mathrm{plant}$ for three years. After fertilization, the soil fertility in eucalyptus forest region will be significantly improved, especially the contents of trace elements such as $\mathrm{N}, \mathrm{P}, \mathrm{K}$, etc.

\subsection{Data}

The experimental data of the data fusion research in this paper are from the soil moisture, soil temperature, $\mathrm{DBH}$ and trunk moisture of eucalyptus plantation at 3 observation points, which are obtained from the sensor network of eucalyptus plantation deployed in Yinling branch of Gaofeng Forest Farm. The three observation points are located in the same small class, and rise 50 meters in turn according to the altitude gradient. One set of microclimate devices in the forest, and the data obtained include environmental temperature, environmental humidity, air pressure, wind speed, wind direction, sunshine, sunshine hours, radiation amount, carbon dioxide concentration, rainfall and other information. Data acquisition time is from July 3, 2019 to now. Please refer to section 3.2 for the networking method of sensor network.

\section{Methods}

\subsection{Holistic approach}

In this paper, the monitoring data fusion research on the Internet of Things of Eucalyptus plantation is taken as a part of the research on the Internet of Things of Eucalyptus plantation. Our method is, in order to continuously observe the environment and tree growth information of Eucalyptus plantation for a long time, deploy the Internet of Things monitoring system, import the data into a cloud management platform that can be stored and analyzed, and conduct multi-level and multiscale data fusion research on this basis to better use the monitoring information.

\subsection{Field Sensor and Network Deployment}

Three node sensor devices are deployed in the Yinling branch of Gaofeng Forest Farm. A total of three sets of soil profile moisture sensors (digital sensors) are respectively connected to 485 interfaces of transmission modules in the three sets of collection nodes, and two sets of self-developed diameter sensors (digital sensors) are connected to 485 interfaces of transmission modules in the two sets of collection nodes. Three sets of trunk runoff sensors and two sets of DBH sensors are analog sensors, which are respectively connected to the collector interfaces in the three sets and two sets of collection nodes, and are sent to the transmission module 485 interface after digital-to-analog conversion by the collector.

A star network based on LoRa protocol is formed between the acquisition node and the gateway, and the gateway is connected with the Internet. LoRa networking communication technology can be used to realize medium and long distance $(300 \mathrm{~m}<$ communication distance $<800 \mathrm{~m}$ ) communication in eucalyptus plantation. LoRa gateway and nodes can form ad hoc networks to realize the aggregation of data collected by nodes. The whole process of sensor measurement, data acquisition, data transmission of acquisition and transmission nodes, gateway data convergence, gateway protocol conversion, MQTT message transmission, cloud MQTT message reception and analysis, and storage work normally.

At the same time, one microclimate observation station and one video monitoring device will be deployed at acquisition point 1 . The positions of the three acquisition points are shown in fig. 1.

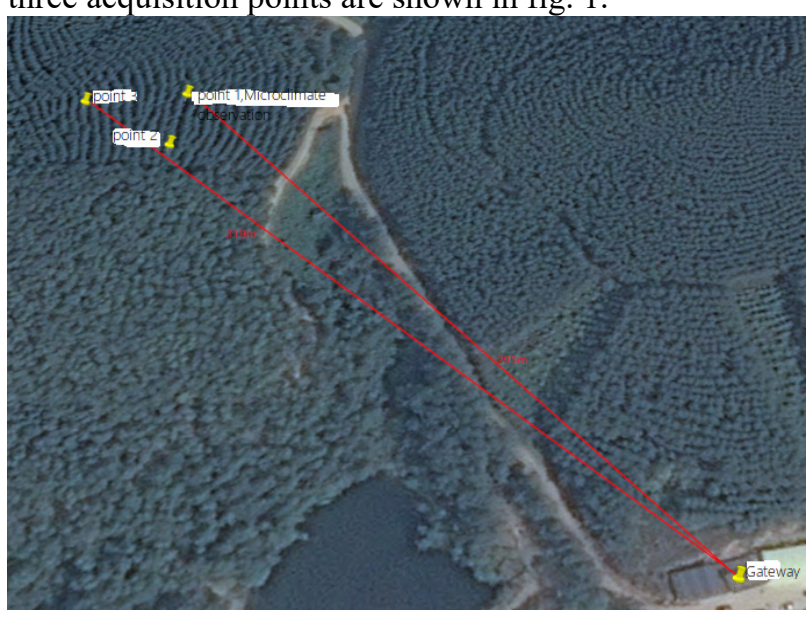

Fig. 1. Sensors in deployment position 


\subsection{Establishment of Monitoring Information Cloud Management System for Eucalyptus Plantation Internet of Things}

Through software and hardware integration, the plantation forest IOT monitoring information cloud management system receives MQTT messages sent by the gateway, completes data analysis and data modeling, data storage management and equipment management, and displays the hardware information and collected data information of the IOT monitoring system. System provides a topology structure of monitoring area, in which the information of the currently accessed collection points and the sensors equipped at each collection point can be seen very clearly. Using the IOT technology of Baidu Cloud[5], monitoring points can be modeled. Based on this model, monitoring data management, retrieval and visual calculation can be uniformly configured and managed.

\subsection{Data Fusion}

This paper only studies the data fusion of single observation index in different time scales.Even though there are still many problems in data fusion, the technical means adopted are different.In this article, we only focus on the fusion of data collected at different time scales for a certain observation index (taking soil water content at different depths as an example).The sensor acquisition frequency is every 10 minutes. How to use these collected data to represent the value of a certain time point in a day, the value of a day, the value of a month, etc. are the issues to be considered in data fusion, and also the data selection problem to be faced first in application analysis. In fact, due to the instability of the sensor network, there will often be data missing in a certain period of time or obvious incorrect data, so it is necessary to use the correct data before and after the automatic data interpolation to improve the availability of data.[3] Because the problems in this area are also complicated, this article will not discuss them.

In this article, if there is missing data or the data is obviously abnormal, we will not make special treatment. Next, we introduce the data fusion method of the same observation point in different time scales. The curve of an observation index in a day is from 0: 00 to 24: 00 , with an interval of 1 hour, all of which are lines for collecting data at intervals of 10 minutes. At the same time, the maximum value and minimum value of this index every 1 hour are given, thus indicating the change range of this value.

The monthly data of the observation indicators are calculated at daily intervals, and the daily value is the average value of the values obtained on the same day. At the same time, the maximum and minimum values of the indicators for each day are given. The maximum and minimum values of each day are the maximum and minimum values of the values obtained on that day.

The annual data of the observation indicators are calculated at monthly intervals, and the monthly value is the average value of the daily value of this month. At the same time, the maximum value and minimum value of each month of the indicators are given. The maximum and minimum values of each month are the maximum and minimum values of the daily values of this month.

The maximum and minimum values under different time scales of the index are given in a general sense to increase the information content of the data, which is helpful to identify erroneous and abnormal data, to understand the changes in eucalyptus plantation growth during the day, month and year, and to facilitate the actual forest management research. In this way, we can obtain the hourly interval data, daily interval data and monthly interval data of a certain observation point from the continuous observation data of the sensor with the frequency of 10 minutes.

\section{Results}

\subsection{Monitoring information acquisition results}

We have established a web-based program to verify the acquisition process of continuous observation data of the entire plantation Internet of Things, and have implemented a time-scale data fusion method. Fig.2 shows the 10-minute data of soil water content at $40 \mathrm{~cm}$ depth obtained from observation point 3 from July to September 2019. Fig.2 shows only one data fragment, and the actual amount of data is very large.

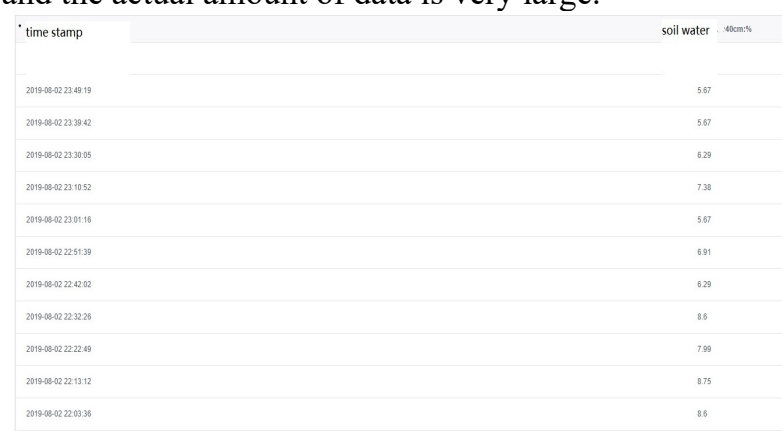

Fig. 2. Sensor data query

\subsection{Data fusion results}

Although the ten-minute soil moisture data have their scientific values, they have no advantages when they need to be analyzed and calculated by using the soil moisture data in days. At this time, it is necessary to use the data fusion method to fuse fine-grained data into coarser time-scale data in days. Fig. 3 shows the soil water content data of $40 \mathrm{~cm}$ underground obtained by observation point 3 on the day of August 2, 2019, which is the result of ten-minute data fusion according to this method. Fig. 4 shows the soil water content data of $40 \mathrm{~cm}$ underground obtained by observation point 3 in August 2019 , which is the result of fusion according to the method in section 3.4. 


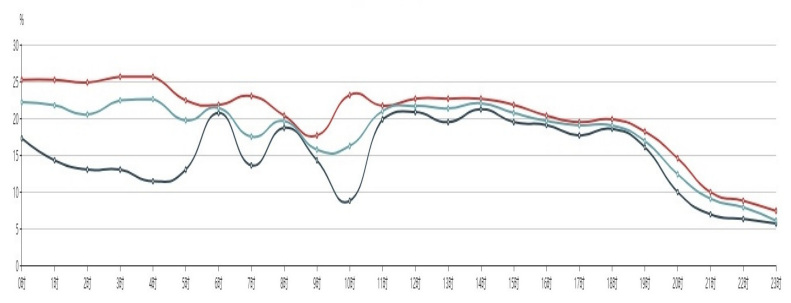

Fig. 3. One-day sensor data fusion

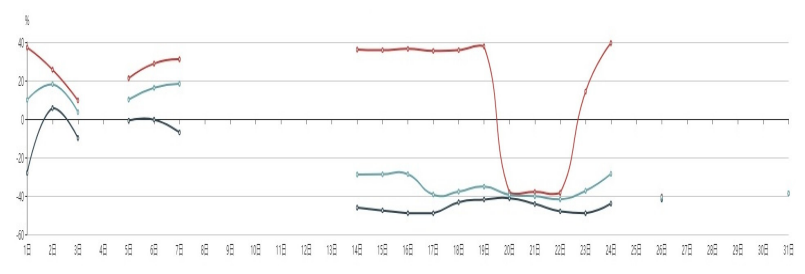

Fig. 4. One-month sensor data fusion

\subsection{Prospect of Data Fusion Application}

In the past 10 years, eucalyptus plantations in Guangxi have developed rapidly, with the area, growth and accumulation of eucalyptus ranking first in the country.However, the argument that eucalyptus is a "water pump", "fertilizer pump" and "green desert" has never stopped. From February 2010 to June 2017, Wildlife Conservation International (FFI) and Stora Enso Company carried out a long-term monitoring project on biodiversity of eucalyptus plantation. Ten young eucalyptus forests and their surrounding buffer zones were randomly selected as monitoring sample plots on the forest subcompartment distribution map, and birds and plants were selected as monitoring indicators.More than 270 species of plants belonging to 209 genera and 84 families are recorded. 109 species of birds were recorded.[6] Continuous long-term observation of soil moisture content and trunk runoff of Eucalyptus plantation will be an important supplement to the above research and will also have various significance.

Minjie Zhu conducted a one-year field experiment in Danzhou forest farm in the western part of Hainan Province. The thermal diffusion probe method was used to measure the stem sap flow of 4-5 years old eucalyptus, and environmental factors were monitored simultaneously to clarify the impact of eucalyptus planting on soil water ecology.[4] Since there is no data transmitted to the cloud after the sensor network is organized, the data can only be collected manually and on time, and the experimental device has been deployed and used for one year. If there is longer-term observation data, there is still much work to be done to study the water consumption of eucalyptus plantation by relevant data fusion methods and environmental climate factors. The research in this paper provides this possibility.

\section{Conclusions}

This research is dramatically speeding up shared information. Historically, forest data have been laborious to gather, and often taking months to distribute. The new network will provide real-time access to environmental sensor data (air temperature, precipitation, relative humidity, wind speed, solar radiation, soil temperature and moisture, stream flow and a webcam to monitor tree phenology) from established research sites to a single point of entry website. Based on the Internet of Things system of Eucalyptus plantation established in Guangxi, this paper discusses the data fusion method of highfrequency monitoring data in different time scales in the face of observation indexes, which is implemented in the program. This fusion method mainly serves for the follow-up research on the relationship between water consumption index of eucalyptus plantation and environment, climate, management, etc. In other words, different research issues may require different data fusion methods, which need further research in the future. Different monitoring indexes of eucalyptus plantations have different fusion methods in time and space, which is our future research direction.

\section{Acknowledgments}

The authors would like to thank the editor and the anonymous reviewers for their comments and suggestions. This work was supported by National Key Research and Development Program of China under Grant No. 2017YFB0504202

\section{References}

1. https://smartforests.org

2. Campbell, John L; Rustad, Lindsey E.; etc.. Quantity is nothing without quality: automated QA/QC for streaming sensor networks [J]. BioScience. 63(7): 574-585 (2013)

3. Wei xiurong. Survey of data fusion in wireless sensor networks [J]. Wireless interconnection technology. 14 (2015)

4. Minjie Zhu. Water Consumption of Eucalyptus Plantation and Its Response to Environmental Factors at Multi-time Scales.[M].Hainan Normal University (2017)

5. Baidu company.Tsdb document (2019)

6. Zhanglei. FFI Analysis on Biodiversity Monitoring Project of Eucalyptus Plantation in Guangxi, Guangxi Forestry, 9 (2017)

7. Barbara J Benson,Barbara J Bond,Michael Hamilton. Perspectives on next-generation technology for environmental sensor networks [J]. Frontiers in Ecology and the Environment 8(4):193-200 (2010)

8. Sun Zhen-wei,Zhao Ping,etc. Seasonal variations of sap flow and transpiration water consumption of introduced tree species Acacia auriculaeformis and 
Eucalyptus citriodora $[\mathrm{J}]$. Chinese Journal of Ecology ,33(10):2588-2595 (2014) 